\title{
The AJ072 antibody against the human transferrin receptor labels the surface and early endosomes of HEK cells by immunofluorescence Claudie Bian ${ }^{1,2}$ \\ ${ }^{1}$ Manufacturing Science and Technologies, Biotech Dpt, Merck, Z.I. de l'Ouriettaz 150, CH-1170, Aubonne, Switzerland ${ }^{2}$ Cell Physiology and Metabolism Dpt, Faculty of Medicine, University of Geneva, 1 rue Michel Servet, CH-1211, Geneva, Switzerland
}

\begin{abstract}
The AJ072 antibody, against the human transferrin receptor, labels the plasma membrane and early endosomes of HEK cells by surface and total immunofluorescence.
\end{abstract}

\section{Introduction}

TfR (Transferrin receptor protein 1, CD71; Uniprot P02786) is a type II transmembrane glycoprotein that binds the iron-carrier glycoprotein transferrin (Tf). Cellular uptake of iron occurs via receptor-mediated endocytosis of diferric Tf/TfR complexes (Candelaria et al., 2021). Here, we describe the ability of the AJ072 recombinant antibody against human TfR to stain the plasma membrane and early endosomes of HEK cells by surface and total immunofluorescence.

\section{Materials \& Methods}

Antibodies: Production of the AJ072 antibody, a miniantibody with the antigen-binding $\mathrm{scFv}$ fused to a rabbit IgG Fc, has been described (Bian, 2022).

Antigen: HEK293T cells were cultured on glass coverslips (Menzel-Gläser, 22×22 $\mathrm{mm}$ ) and grown in DMEM GlutaMAX ${ }^{\mathrm{TM}}$ (Gibco 31966) supplemented with 10\% Fetal Bovine Serum (Gibco 10270) and $100 \mu \mathrm{g} / \mathrm{mL}$ of penicillin-streptomycin (Gibco 15070-063) at $37^{\circ} \mathrm{C}$ and $8 \% \mathrm{CO}_{2}$.

Protocol: Cells were incubated with preheated DMEM GlutMAX $^{\mathrm{TM}}$ serum-free for $10 \mathrm{~min}$ at $37^{\circ} \mathrm{C}$. Cells were then incubated with transferrin AlexaFluor ${ }^{\mathrm{TM}}-546$ conjugate (ThermoFisher T23364) at $5 \mu \mathrm{g} / \mathrm{mL}$ in DMEM GlutMAX ${ }^{\mathrm{TM}}$ for $30 \mathrm{~min}$ at $37^{\circ} \mathrm{C}$. Then on ice, cells were rinsed thrice with cold PBS $+0.2 \%(\mathrm{w} / \mathrm{v})$ BSA (PBSBSA). Cells were then incubated with undiluted AJ072 for 30 min at $4{ }^{\circ} \mathrm{C}$, rinsed thrice with cold PBS-BSA and incubated for $30 \mathrm{~min}$ at $4{ }^{\circ} \mathrm{C}$ with secondary goat antirabbit $\mathrm{IgG}$ conjugated to AlexaFluor ${ }^{\mathrm{TM}}-488$ (1:400, Thermo Fisher A11034). Cells were then rinsed thrice with cold PBS-BSA (3 $\mathrm{min}$ ) and once with cold PBS (3 $\mathrm{min})$. At room temperature, cells were then fixed with $\mathrm{PBS}$ $+4 \%$ paraformaldehyde (w/v) (Applichem A3013) for 30 min and blocked with PBS $+40 \mathrm{mM}$ ammonium chloride
$\left(\mathrm{NH}_{4} \mathrm{Cl}\right)$ (Applichem A3661). Cells were then permeabilized in PBS containing $0.2 \%(\mathrm{w} / \mathrm{v})$ saponin (Sigma S7900) for $5 \mathrm{~min}$, washed once (3 min) with PBSBSA, and incubated for $30 \mathrm{~min}$ with undiluted AJ072. After 3 washes (3 min) with PBS-BSA, cells were incubated for $30 \mathrm{~min}$ with secondary goat anti-rabbit IgG conjugated to AlexaFluor ${ }^{\mathrm{TM}}$-647 (1:400, Thermo Fisher A21245), then washed thrice with PBS-BSA and once with PBS, and mounted on slides (Menzel-Gläser, 76×26 $\mathrm{mm})$ with Möwiol (Hoechst) $+2.5 \%(\mathrm{w} / \mathrm{v})$ DABCO (Fluka 33480). For the control cells, no primary antibody AJ072 was used. Pictures were taken using a Zeiss LSM800 Airyscan confocal microscope (Bioimaging Core Facility, Faculty of Medicine, University of Geneva), with a Plan-APO 40x/1.4 Oil DIC (UV) VIS-IR objective.

\section{Results}

Using a cell surface labeling protocol, AJ072 successfully labeled the plasma membrane (Fig. 1, upper panel, green channel, arrows), as previously described (Bian, 2022). Using a total cell labeling protocol, AJ072 also labeled early endosomes in HEK cells (Fig. 1, upper panel, red channel, arrowheads), as indicated by co-localization with transferrin, a known marker for early endosomes (Fig. 1, upper panel, gray channel, arrowheads). No staining was observed when the primary antibody was omitted (Fig. 1, lower panels, green and red channels).

\section{References}

Bian C. The AJ072 antibody against the human transferrin receptor labels HeLa cells by surface immunofluorescence. Antibody Reports, 2022, 5:e644. doi:10.22450/ journals/abrep.2022.e644.

Candelaria PV, Leoh LS, Penichet ML, Daniels-Wells TR. Antibodies targeting the Transferrin Receptor 1 (TfR1) as direct anti-cancer agents. Front Immunol. 2021; 12:607692. PMID: 33815364.

\section{Conflict of interest}

The authors declare no conflict of interest. 

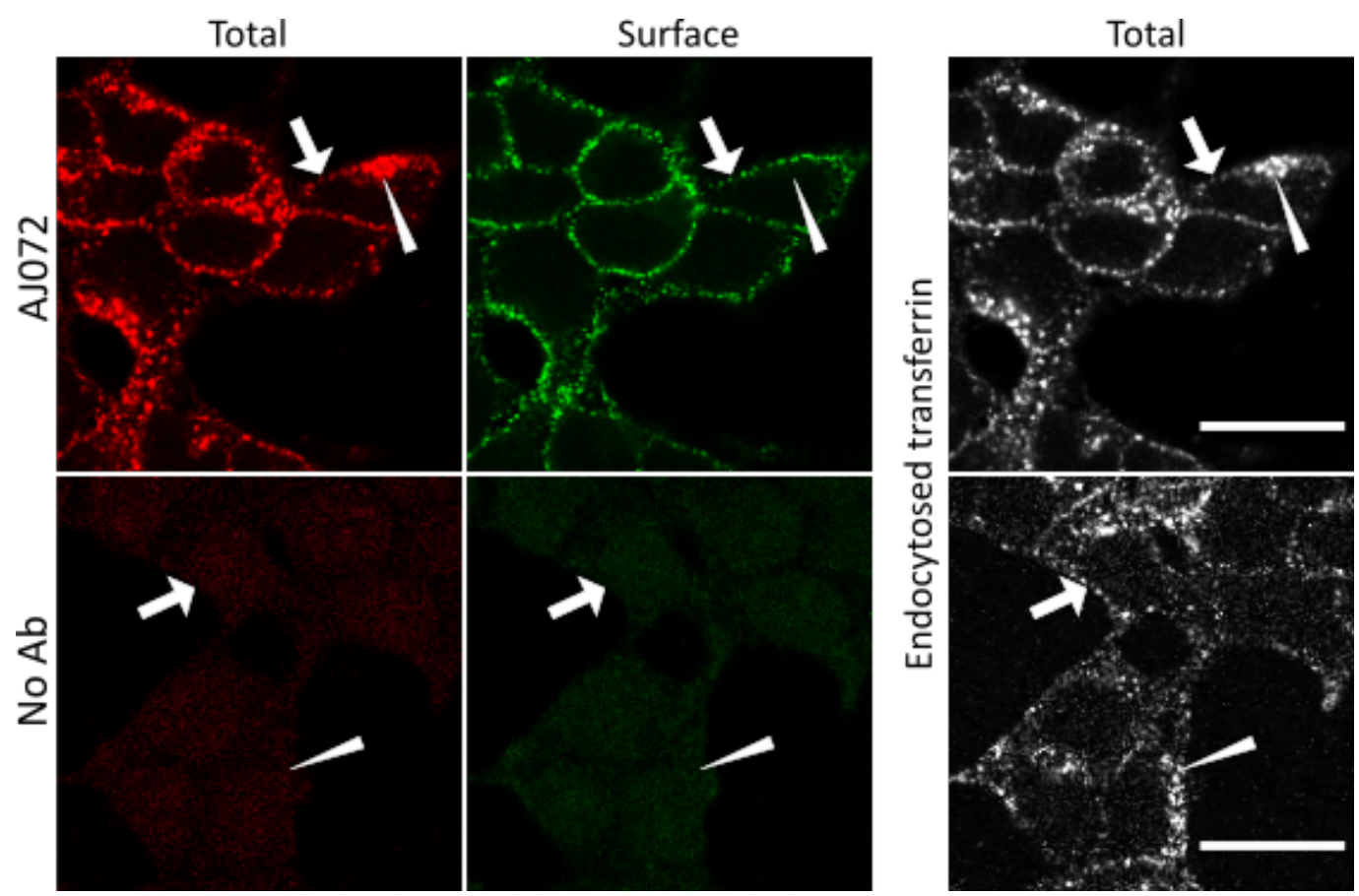

Fig. 1. AJ072 successfully labeled the cell membrane (green channel, arrows) of HEK cells by surface immunofluorescence. AJ072 also successfully labeled the plasma membrane (arrows) and early endosomes (arrowheads) of HEK cells by total immunofluorescence. Early endosomes and cell surface were stained with surface-bound and endocytosed transferrin Alexa Fluor ${ }^{\mathrm{TM}}-546$ (in gray). The AJ072 intracellular labeling co-localized with the endocytosed transferrin (arrowheads). No labeling was observed when the primary antibody was omitted (No Ab panels). Scale bar: $20 \mu \mathrm{m}$. 